\title{
THE VALUE OF NECROPSY IN QUALITY CONTROL OF MEDICAL DIAGNOSIS - THE GOLD STANDARD FOR YEARS TO COME
}

\author{
doi: $10.1590 / \mathrm{S} 1807-59322009000300002$
}

Paulo Sampaio Gutierrez, Thales de Brito, Maria Beatriz Sampaio Lopes, Venancio Avancini Ferreira Alves

Despite the well-known valuable role of necropsies in pathology training and their indisputable importance for quality assurance in medical practice, the number of necropsies performed worldwide has decreased over the last few years. This has been ascribed, in part, to the idea that, at the time of death, most diagnoses have already been made using new diagnostic technologies, including clinical, morphological, and molecular pathology and imaging methods. In CLINICS 2008;63(5):581-8, Kotovicz et al. ${ }^{1}$ compared necropsy and clinical diagnosis at our large teaching hospital, where, although still performed, the number of necropsies has also declined. Unexpectedly, major missed diagnoses, which could have led to a change in management with increased survival or cure, were detected in $16.3 \%$ of cases. Minor diagnoses not directly related to the cause of death were detected in an additional $28.1 \%$ of cases. In a similar study at the Heart Institute of the same hospital, Saad et al. ${ }^{2}$ also found major disagreements regarding the cause of death in $30.0 \%$ of cases in a sequential series of 406 necropsies, mostly involving cardiovascular and respiratory diseases. In both articles, the authors quoted other studies from different hospitals and countries, and found that, although new technological tools had indeed diminished the differences between clinical and necropsy findings, major diagnostic discrepancies were found in $14-39 \%$ of cases, even in recent series from developed countries ${ }^{3-6}$. These papers highlight the occurrence of remarkable diagnostic failures, with important consequences for patient management.

One reason for the decline in autopsy rates seems to be family concern about the procedure. In the United Kingdom, this concern arose mainly after the revelation of unauthorized organ retention ${ }^{7}$. In Brazil, and possibly in other countries, there is a cultural desire that burials occur promptly, and families will not often consent to necropsies because of the delay caused by the procedure. Given this, pathology depart- ments should try to decrease such delays as much as possible. However, at the Heart Institute, where for several years families were asked to consent to an autopsy upon hospital admission, the percent of family authorization $(\sim 60 \%)$ was much higher than the actual necropsy rate $(\sim 25 \%)$, indicating that, in many cases, even when consent is granted, physicians did not request a necropsy.

Thus, it seems reasonable to question what has caused this lack of physician interest. Some reasons might include: a desire to avoid ethical or legal problems that might arise if any kind of error or disagreement was found between the clinical and necropsy findings, procedural costs, or the perception that, with the presently available technology, there is little to be revealed by postmortem examination. Although this last idea is clearly contradicted by the articles of Kotovicz et al., ${ }^{1}$ Saad et al., ${ }^{2}$ and others quoted in those studies, it deserves further consideration.

Necropsy results extend beyond individual cases. Even when one necropsy does not reveal any new findings, it may be performed as part of a study and thereby help identify morphological traits of diseases not readily apparent in individual patients. This scenario is exemplified by the fact that most cases of cardiac amyloidosis are still diagnosed only at necropsy ${ }^{8}$. Furthermore, despite advances in image analysis and other methods, pulmonary embolism, endocarditis, and the initial phases of bronchopneumonia cannot currently be definitively diagnosed using imaging techniques alone $e^{7,8}$. Virtopsy ${ }^{9}$, which is a method of postmortem examination involving magnetic resonance imaging or tomography studies of a corpse, along with needle sampling, would probably also be of little help in many cases.

In a recent comment on the reasons behind the apparent diminution by physicians of the importance of necropsy, Pompilio and Vieira ${ }^{10}$ stated that this loss of interest might be related to the increasing influence of technological tools 
in the practice of medicine. Indeed, the very concept of disease may be changing. In the past, nearly all conditions, except psychiatric disorders, were defined based on morphological pathology. Today, our understanding of disease and disease treatment is further enhanced by information related to genetic polymorphisms, cell counting, gene expression profiling, proteomics, and a type of "new-humoral" medicine involving knowledge concerning cytokines and many other circulating molecules. Most of these new techniques should be major tools in the modern pathology laboratory, with each approach contributing to a more complete concept of disease. For example, a single disease might be linked to a number of different genetic polymorphisms affecting the production, destruction, or pre- or post-translational modification of a single protein. Similarly, the term "inflammatory" infiltrate is no longer sufficient; the effect of such an infiltrate can depend on the subtype and functional status of each cell present, as some cell types modulate inflammation in a number of ways.

On the other hand, imaging tools represent a large proportion of newly available instruments. Their increasing importance reinforces the fact that morphologic pathology is still pivotal to diagnosis and to the definition of many diseases. Except for situations in which movements or flows are required, all of these instruments attempt to examine, while the patient is still alive, alterations in human organs and cells that were previously detected only at necropsy. This suggests that necropsy remains the gold standard for diagnosis. For instance, the term "virtual histology" appears in 77 articles found via PubMed, 64 concerning intravascular ultrasound, 10 on confocal laser endoscopy of the gastrointestinal tract, and 3 relating to other organs. As demonstrated in the article by Mauad et al. ${ }^{1}$, as well as others, significant differences still exist between virtual and real pathology. Therefore, in our opinion, teaching hospitals should strive to perform necropsies for $100 \%$ of in-hospital deaths. Only family denial should prevent the performance of these procedures, and efforts should be made to decrease the number of such cases.

In conclusion, we believe that improved accuracy of new diagnostic methods could be achieved through closer collaboration between clinicians, radiologists, and clinical and surgical pathologists. In many hospitals, including Hospital das Clínicas-FMUSP, such integration is rapidly growing within most sub-specialties (the most notable being fine-needle aspiration), yielding, in certain clinical contexts, major improvements in accuracy. Within this type of framework, necropsies will remain the gold standard for the multi-specialty approach to diagnosis and will guide us in the safe and optimal use of the present and future diagnostic armamentarium.

\section{REFERENCES}

1. Kotovicz F, Mauad T, Saldiva PHN. Clinico-pathological discrepancies in a general university hospital in São Paulo, Brazil. Clinics. 2008;63(5):581-8.

2. Saad R, Yamada AT, Rosa FHP, Gutierrez PS, Mansur AJ. Comparison between clinical and autopsy diagnoses in a Cardiology hospital. Heart. 2007;93:1414-9.

3. Sonderegger-Iseli K, Burger S, Muntwyler J, et al. Diagnostic errors in three medical eras: a necropsy study. Lancet. 2000;355:2027-31.

4. Perkins GD, McAuley DF, Davies S, et al. Discrepancies between clinical and postmortem diagnoses in critically ill patients: an observational study. Crit Care. 2003;7:129-32.

5. Combes A, Mokhtari M, Couvelard A, et al. Clinical and autopsy diagnoses in the intensive care unit: a prospective study. Arch Intern Med. 2004;164:389-92.
6. Klock C, Cerski M, Goldani LZ. Histopathological Aspects of Neurocryptococcosis in HIV-Infected Patients: Autopsy Report of 45 Patients. Int J Surg Pathol. 2008 Jul 8. [Epub ahead of print].

7. Aiello VD, Debich-Spicer D, Anderson RH. Is there still a role for cardiac necropsy in 2007? Cardiol Young. 2007;17(Suppl 2):97-103.

8. Gutierrez PS, Fernandes F, Mady C, Higuchi ML. Clinical, electrocardiographic and echocardiographic findings in significant cardiac amyloidosis detected only at necropsy: comparison with cases diagnosed in life Arq Bras Cardiol. 2008;90:191-6.

9. Jackowski C, Schweitzer W, Thali M, et al. Virtopsy: postmortem imaging of the human heart in situ using MSCT and MRI. Forensic Sci Int. 2005;149:11-23.

10. Pompílio CE, Vieira JE. The technological invention of disease and the decline of autopsies. Sao Paulo Med J. 2008;126:71-2. 